\title{
ELECTRÓLISIS PERCUTÁNEA INTRATISULAR (EPI®)
}

\section{M. a Soledad Pérez Antoñanzas}

Servicio de Cirugía Ortopédica y Traumatología

Hospital Universitario Príncipe de Asturias. Alcalá de Henares. Madrid

\section{Introducción}

La electrólisis percutánea intratisular $\left(E P{ }^{\circledR}\right)$ es una técnica de fisioterapia, mínimamente invasiva, de reciente desarrollo, que consiste en aplicar localmente sobre un tejido degenerado una corriente galvánica, a través de una aguja de acupuntura ecoguiada, que actúa como electrodo negativo (cátodo). Sus efectos iónicos provocan una reacción electroquímica (ablación electrolítica no termal), que induce una respuesta inflamatoria controlada, permitiendo activar los mecanismos celulares implicados en la fagocitosis y en la regeneración del tejido blando dañado (tendón, ligamento, músculo, etc. $)^{(1-3)}$.

El creador de esta técnica es el fisioterapeuta José Manuel Sánchez Ibáñez, que la desarrolló y publicó inicialmente en su tesis doctoral Evolución clínica en el tratamiento de la entesopatía rotuliana crónica mediante electro-estimulación percutánea ecodirigida: estudio de una serie de casos en población deportiva (Facultad de Veterinaria de la Universidad de León, 2013). En ella expone los efectos terapéuticos de la aplicación de una corriente galvánica, dirigida exclusivamente al área de tendón degenerado. Dicha corriente provoca una reacción tisular que induce una rápida reparación de dicho tendón, en un estudio experimental en animales, junto a una serie de casos clínicos en deportistas con tendinopatía rotuliana(4).

Aunque inicialmente la técnica fue descrita y estudiada para el tratamiento de la tendinopatía rotuliana, posteriormente su uso se ha extendido a otras patologias musculotendinosas e incluso a patologias por atrapamiento de nervios periféricos.

Esta técnica también se denominaba microrregeneración endógena guiada (MEG), pero actualmente se utiliza únicamente el término EPI ${ }^{\circledR}$. 


\section{¿Qué dice la literatura?: revisión bibliográfica y búsqueda de evidencia}

A pesar de que existe una experiencia de más de 15 años en el uso de la técnica EPI ${ }^{\circledR}$ y de haberse extendido su uso entre los clubes deportivos en todo el mundo, ha sido en los últimos años cuando ha aparecido literatura científica avalando esta técnica. Estudios recientes apoyan sus buenos resultados clínicos en el tratamiento de la tendinopatía degenerativa del tendón rotuliano, en comparación con el tratamiento conservador $r^{(5-7)}$.

Existe evidencia científica en estudios de investigación en animales. Abat et al. concluyeron, con un nivel de evidencia I, que la técnica EPI ${ }^{\circledR}$ produce, en la lesión tendinosa inducida mediante inyección de colagenasa de tipo I en ratas, un aumento de los mecanismos moleculares antiinflamatorios y angiogénicos ${ }^{(7)}$. Sin embargo, el propio Sánchez Ibáñez manifiesta, en su anteriormente mencionada tesis doctoral, que "existen controversias respecto a la validez de los estudios acerca de tenopatías en modelos animales"(4).

En estudios experimentales con tendones humanos se ha visto una desproporción de ciertas citocinas y metaloproteasas (MMP), prostaglandinas E2 (PGE2), interleucinas-6 (IL-6) e interleucinas-1b (IL-1b), responsables y mediadores en la respuesta inflamatoria ${ }^{(8,9)}$.

Otros estudios experimentales recientes demuestran que el uso de EPI ${ }^{\circledR}$ en la tendinopatía patelar aumenta las proteínas antiinflamatorias, como el factor de transcripción nuclear (receptor de la superfamilia de los receptores esteroideos) y el receptor PPAR-y (peroxisome proliferator activated receptor gamma), que tienen un papel primordial en la inhibición de la expresión de moléculas proinflamatorias secretadas por los macrófagos, así como el factor de necrosis tumoral TNF- $\alpha$ (tumor necrosis factor) e interleucinas IL-6 e IL-1 $\beta^{(7)}$.

Por este mecanismo, se genera una respuesta molecular en el tejido tratado, altamente beneficiosa en el transcurso de una tendinopatía. En el estudio se objetivó un aumento de la expresión de las proteínas citocromo
C (capaz de activar las últimas fases de la apoptosis en las tendinopatías), de la Smac/Diablo (proteína mitocondrial que induce la apoptosis), del factor de crecimiento endotelial vascular (vascular endothelial growth factor, VEGF), proteína señalizadora implicada en la angiogénesis y la vasculogénesis, y de su receptor VEGFR-2 (vascular endotelial growth factor receptor-2), que actúa como mediador más importante de la angiogénesis. La literatura identifica los receptores VEGFR-1 y VEGFR-2 como los expresados sobre todo en el tendón de Aquiles humano ${ }^{(10)}$.

Los resultados de Abat et al. demuestran un aumento del VEGFR-2 tras el tratamiento con la técnica $E P I{ }^{\circledR}$, lo que evidencia una modificación en la vía de apoptosis celular y un aumento de la angiogénesis ${ }^{(7)}$ (Tabla 1).

Según los trabajos de Sánchez Ibáñez, la EPI ${ }^{\circledR}$, dirigida a la zona afectada del tendón rotuliano en su inserción, sería capaz de modificar la situación local del tendón y de poner en marcha un proceso de reparación ${ }^{(4)}$.

A pesar de la evidencia científica en los estudios experimentales sobre los efectos beneficiosos de la $E P{ }^{\circledR}$, en la activación de los mecanismos moleculares y celulares tendinosos responsables de la fagocitosis y regeneración del tejido degenerado, en la revisión bibliográfica realizada no

\section{Tabla 1. EFECTOS FISIOLÓGICOS DE LA TÉCNICA EPI®}

Disminución de citocinas inflamatorias (IL-10-, TNF- $\alpha$, etc.)

Efectos antiinflamatorios

Facilitación de las células fagocíticas al foco de la lesión

Activación de proteínas regenerativas del tendón y músculo (Ppar- $\delta$, Scx, Col-1, etc.)

Aumento del riego sanguíneo

Disminución de neurotransmisores nociceptivos y del dolor

Activación de los mecanismos de regeneración de los tejidos blandos lesionados

Restablecimiento del movimiento de los fluidos de la matriz extracelular

Efectos antifibróticos del tejido conectivo

Estimulación de la síntesis de colágeno de tipo 1

Estimulación de la revascularización

Aumento de la $\mathrm{pO}_{2}$ tisular y del $\mathrm{pH}$ del tendón degenerado

Restablecimiento de la homeostasis del tejido blando degenerado 
aparece ningún metaanálisis, ni ensayo clínico, ni estudios clínicos con nivel de evidencia I ni II en pacientes tratados con esta técnica.

Las publicaciones científicas sobre EPI ${ }^{\circledR}$ hacen referencia a series de casos clínicos o a propósito de un caso único. Son estudios observacionales descriptivos, no aleatorizados, con escaso nivel de evidencia. No existen estudios comparativos con otras técnicas ni frente a pacientes control. Valera et al. han publicado buenos resultados en tendinopatía rotuliana crónica con EPI ${ }^{\circledR}$ asociada a trabajo excéntrico y estiramientos, con el $80 \%$ de pacientes dados de alta tras 6 sesiones de tratamiento ${ }^{(11)}$. En la mayoría de las publicaciones y comunicaciones científicas aparece, como autor principal o colaborador, el creador de la técnica, que también ha comunicado buenos resultados en pacientes diagnosticados de epicondilitis en una serie de 40 pacientes en publicaciones a través de la plataformas virtuales de fisioterapia ${ }^{(12)}$.

Los autores de los principales trabajos declaran conflicto de intereses, ya que uno de los autores posee la patente de los dispositivos para la técnica EPI ${ }^{\circledR}$ o es el creador de la técnica.

\section{Indicaciones de la terapia}

La EPI ${ }^{\circledR}$ es una técnica originariamente enfocada para el tratamiento de las tendinosis (tendinitis evolucionadas a la cronicidad), en las cuales los fenómenos inflamatorios han evolucionado a una degeneración del tendón. No obstante, actualmente se está enfocando al tratamiento de una amplia variedad de lesiones en tejidos blandos.

Aunque inicialmente la técnica fue descrita y estudiada para el tratamiento de la tendinopatía rotuliana, posteriormente su uso se ha extendido a otras patologías musculotendinosas de miembro inferior. Asimismo, han sido publicados, con buenos resultados, un caso clínico de tratamiento sintomático de un paciente diagnosticado de sindrome del túnel del carpo severo, con mejoría sintomática y neurofisiológica ${ }^{(13)}$, y otro de epicondilalgia ${ }^{(14)}$, igualmente con mejoría clínica.

Actualmente, la técnica EPI ${ }^{\circledR}$ se aplica en el tratamiento de tendinopatías, fascitis plantares, bursitis, contracturas musculares, fibrosis musculares, puntos gatillo miofasciales, roturas de fibras, esguinces, atrapamientos nerviosos, entesopatías y periostitis (Tabla 2 ).

Las patologías tratadas más frecuentemente a nivel de pie y tobillo son las tendinopatías aquíleas, fascitis plantares y la patología conocida como tennis-leg (rotura de la porción distal del gemelo interno), si bien también se aplica en 


\begin{tabular}{|l|}
\hline Tabla 3. CONTRAINDICACIONES ABSOLUTAS \\
\hline Linfedemas generalizados y severos de las extremidades \\
\hline Bacteriemia \\
\hline Artritis sépticas \\
\hline Alteraciones de la coagulación \\
\hline Gestación \\
\hline Psoriasis \\
\hline Procesos oncológicos \\
\hline Cardiopatías \\
\hline Marcapasos \\
\hline Osteosíntesis y prótesis metálicas \\
\hline Osteoporosis \\
\hline Fracturas osteocondrales \\
\hline
\end{tabular}

\section{Tabla 4. CONTRAINDICACIONES RELATIVAS}

\begin{tabular}{|l|}
\hline Fibromialgia \\
\hline Neuroestimuladores epidurales \\
\hline Depresión \\
\hline Hematomas \\
\hline Heridas \\
\hline Tromboflebitis \\
\hline Epilepsia \\
\hline Isquemia \\
\hline SDRC (síndrome dolor regional complejo) \\
\hline
\end{tabular}

lesiones ligamentarias y tendinopatías del tendón tibial posterior y de los tendones peroneos.

La técnica EPI ${ }^{\circledR}$ está contraindicada principalmente en pacientes con tumores, con infección articular o sistémica, y con alteraciones de la hemostasia. Las contraindicaciones absolutas y relativas de esta técnica se muestran en las Tablas 3 y 4.

\section{Técnica de aplicación}

Debido a que la aplicación de la técnica EPI ${ }^{\circledR}$ es dolorosa, como consecuencia de la reacción elec- troquímica directa que se produce sobre el tendón, se recomienda administrar sobre la piel de la zona a tratar un anestésico local de uso tópico a base de lidocaina al 4\%, 30 minutos antes de la EPI ${ }^{\circledR}$. La ecografía permite localizar la región a anestesiar. Además, se puede utilizar crioanalgésico tópico en el momento de la punción.

La corriente galvánica se aplica mediante una aguja de acupuntura estéril, de un solo uso, de calibre de 0,32 mm y $25 \mathrm{~mm}$ de longitud. El electroestimulador suministra una corriente máxima de $30 \mathrm{~mA}$ y dispone de un aplicador (manípulo) con un porta-agujas.

Siguiendo los criterios propuestos por el autor (Sánchez-Ibáñez, 2005), para el tendón rotuliano se realizan 3 intervenciones por zona (un total de 9): interfase superficial (paratendón), intratendón y profunda. Se aplica habitualmente una corriente continua en el rango de seguridad de 2 a $4 \mathrm{~mA}$, $20 \mathrm{~V}$ y $0,239 \mathrm{~J}$, durante un tiempo de $4 \mathrm{~S}$ por punción, con una duración total de 36 s de aplicación de la $E P I^{\circledR(4)}$.

Habitualmente se realiza una sesión cada 7 días. Suele aparecer mejoría del dolor habitualmente a las 3-4 sesiones. En caso de persistir la clínica, en ocasiones se han ampliado hasta 1011 sesiones.

\section{Resultados de la literatura}

Se han documentado resultados satisfactorios en tendinopatía rotuliana evaluando los efectos terapéuticos de la EPI ${ }^{\circledR}$ mediante el test VISA-P (Victorian Institute of Sport Assessment-Patella score), el test de Tegner y el test de Roles y Maudsley, con mejoría clínica y funcional, con un seguimiento de 10 años ${ }^{(5)}$. Los pacientes fueron tratados de forma conjunta mediante EPI ${ }^{\circledR}$ guiada por ecografía y ejercicios excéntricos, obteniendo una amplia mejoría de la función de la rodilla y un rápido retorno al nivel de actividad previo.

La EPI ${ }^{\circledR}$ produce una rápida regeneración del tejido degenerado, lo que conduce a la producción de nuevas fibras de colágeno inmaduro que se transforma en maduro mediante estímulos excéntricos. De este modo, se obtienen buenos resultados en un seguimiento de 2 años, con mejoría funcional y del dolor según los estudios de Abat et al.(6). 
Valera-Garrido et al. publican un estudio observacional en 32 pacientes a los que se les aplicaba EPI ${ }^{\circledR}$ asociada a trabajo excéntrico y estiramientos evaluando los resultados mediante la escala VISA-P(11). Al final del tratamiento se apreciaron cambios importantes en el análisis de la funcionalidad; la puntuación media obtenida en el total de 32 sujetos con la escala VISA-P fue de 80,0 puntos (DE: 12) (27 puntos más que al inicio).

Sánchez-lbáñez et al. realizan un estudio prospectivo de 39 pacientes afectos de tendinopatía aquílea, tratados mediante terapia EPI ${ }^{\circledR}$ en asociación con ejercicios excéntricos y evaluados mediante VISA-A, y publican resultados estadísticamente significativos de mejoría clínica en cuanto al dolor y la función a los 3 meses ${ }^{(15)}$.

También se han publicado resultados positivos en estudios prospectivos con mejoría clínica en casos de epicondilitis tratadas con EPI ${ }^{\circledR}$ asociados ejercicios excéntricos y estiramientos ${ }^{(16)}$.

\section{Discusión}

El tratamiento de las tendinopatías es un tema de debate frecuente en medicina deportiva y fisioterapia, habiéndose descrito numerosas técnicas de tratamiento sin llegar hoy en día a un consenso sobre cuál es la óptima.

La EPI ${ }^{\circledR}$ es una técnica novedosa en el campo de la fisioterapia que parece demostrar resultados prometedores en las tendinopatías crónicas. Pero el número de artículos publicados es escaso, perteneciendo en su mayor parte a un reducido grupo de autores, entre los que se incluye el propio autor de la técnica y sus colaboradores y/o compañeros en sus centros de trabajo.

La $E P{ }^{\circledR}$ actúa sobre el área afecta poniendo en marcha el proceso biológico de reparación del colágeno, aumentando los mecanismos moleculares antiinflamatorios y angiogénicos. El aumento de la funcionalidad, que parece conseguirse rápidamente, no se ve reflejado en los cambios en la propia estructura del tendón (engrosamiento, imágenes hipoecogénicas) que tardan más en producirse, precisando de un proceso biomecánico de remodelación mediante excéntricos y estiramientos.

De todos los artículos revisados y publicados actualmente, ninguno refleja de forma definitiva que la $E P{ }^{\circledR}$ por sí misma, como tratamiento aislado, sea efectiva como tratamiento de las tendinopatías. La mayoría son estudios observacionales y retrospectivos, o experimentales en animales. Por otra parte, los estudios clínicos con pacientes combinan el tratamiento con EPI ${ }^{\circledR}$ con un programa de trabajo excéntrico en la mayoría de los casos, sin existir estudios comparativos entre asociación o no de terapias y/o que incluyan casos y controles.

Al igual que ocurre con el resto de los tratamientos actualmente disponibles para las tendinopatías, no existe evidencia que apoye la EPI ${ }^{\circledR}$ como técnica de elección. Son necesarios ensayos clínicos amplios, aleatorizados y controlados, además de estudios comparativos entre las diferentes técnicas actualmente disponibles, para poder determinar el tratamiento de elección para esta patología ${ }^{(17)}$.

\section{Conclusiones}

En la actualidad existen variedad de técnicas de tratamiento de las tendinopatías. La EPI ${ }^{\circledR}$ es una técnica de fisioterapia, mínimamente invasiva, dolorosa en su aplicación, utilizada principalmente en el tratamiento de las tendinopatías y entesopatías.

Según estudios experimentales, a través de su mecanismo de acción se produce una modificación de los mecanismos moleculares antiinflamatorios y angiogénicos del tejido colágeno degenerado, induciendo la reparación del mismo.

La efectividad del tratamiento, según sus autores, llega a ser del 80-85\%, siempre que se aplique de forma ecoguiada y con una correcta indicación. El número de sesiones medio suele ser de 4 , aunque con una sola aplicación ya se pueden conseguir resultados favorables.

Sin embargo, no existe actualmente evidencia científica clínica que aporte validez a su uso como "patrón de oro" o técnica de elección en el tratamiento de las tendinopatías, siendo necesarios estudios aleatorizados y controlados de esta técnica, así como estudios comparativos con otras técnicas para determinar su eficacia terapéutica y/o superioridad sobre otras terapias en el tratamiento de las tendinopatías, aunque podría ser recomendable en asociación con otras técnicas. 


\section{Bibliografía}

1. Sánchez Ibáñez J, Fernández M, Moreno C, Marti D, Belón P. Ultrasound-Guided EPI ${ }^{\circledR}$ Technique and Eccentric Exercise, New Treatment for Achilles and Patellar Tendinopathy Focused on the Region-Specific of the Tendon. Orthop Muscular Syst. 2015;4(4):1-6.

2. Valera Garrido F. Comparison Of The Acute Inflammatory Response And Proliferation Of Dry Needling And Electrolysis Percutaneous Intratissue (EPI ${ }^{\circledR}$ ) In Healthy Rat Achilles Tendons. Br J Sports Med. 2013;47(9):29.

3. Sánchez-Ibáñez JM, Colmena C, Benabent J, Garcia-Herreros S, Valles SL. New Technique in Tendon Sport Recovery. Percutaneous Electrolysis Intratissue (EPI $\left.{ }^{\circledR}\right)$. Int J Phys Med Rehabil. 2013;1:113.

4. Sánchez-Ibáñez JM. Evolución clínica en el tratamiento de la entesopatía rotuliana crónica mediante electroestimulación percutánea ecodirigida: estudio de una serie de casos en población deportiva. Tesis Doctoral, Universidad de León; 2013.

5. Abat F, Gelber P, Polidori F, Monllau JC, Sánchez-Ibáñez JM. Clinical results after ultrasound-guided intratissue percutaneous electrolysis $\left(E P{ }^{\circledR}\right)$ and eccentric exercise in the treatment of patellar tendinopathy. Knee Surg Sports Traumatol Arthrosc. 2015;23:1046-52.

6. Abat F, Diesel WJ, Gelber PE, Polidori F, Monllau JC, Sánchez-Ibáñez JM. Effectiveness of the Intratissue Percutaneous Electrolysis $\left(\mathrm{EPI}^{\circledR}\right)$ technique and isoinertial eccentric exercise in the treatment of patellar tendinopathy at two years follow-up. Muscles Ligaments Tendons J. 2014;4:188-93.

7. Abat F, Valles SL, Gelber PE, Polidori F, Stitik TP, García-Herreros S, et al. Mecanismos moleculares de reparación mediante la técnica electrólisis percutánea intratisular en la tendinosis rotuliana. Rev Esp Cir Ortop Traumatol. 2014;58(4):201-5.

8. Skutek M, van Griensven M, Zeichen J, Brauer N, Bosch $U$. Cyclic mechanical stretching enhances secretion of Interleukin 6 in human tendon fibroblasts. Knee Surg Sports Traumatol Arthrosc. 2001;9:322-6.
9. Tsuzaki M, Bynum D, Almekinders L, Yang X, Faber J, Banes AJ. ATP modulates load-inducible IL-1beta, COX 2 , and MMP-3 gene expression in human tendon cells. J Cell Biochem. 2003;89:556-62.

10. Petersen W, Pufe T, Zantop T, Tillmann B, Tsokos M, Mentlein R. Expression of VEGFR-1 and VEGFR-2 in degenerative Achilles tendons. Clin Orthop Relat Res. 2004:420:286-91.

11. Valera-Garrido F, Minaya-Muñoz F, Sánchez-Ibáñez JM. Efectividad de la electrólisis intratisular (EPI $\left.{ }^{\circledR}\right)$ en las tendinopatías crónicas del tendón rotuliano. Trauma. 2010;21(4):227-36.

12. Sánchez-Ibáñez JM. Epicondilitis o codo de tenista. Terapia acelerada mediante microrregeneración endógena guiada (MEG). 2009 Disponible en: https:// www.efisioterapia.net/articulos/epicondilitis-o-codo-del-tenistaterapia-acelerada-microregeneracion-endogena-guiada-meg.

13. Valera F, Minaya F, Sánchez-Ibáñez JM. Cambios clínicos en el síndrome del túnel del carpo con la aplicación de la electrólisis percutánea intratisular $\left(E P I{ }^{\circledR}\right)$. Fisioter Calid Vida. 2011;14(1):13-6.

14. Minaya F, Valera F, Sánchez-lbáñez JM. Uso de la Electrólisis Percutánea Intratisular $\left(\mathrm{EPI}^{\circledR}\right)$ en la Epicondilagia Crónica: Caso clínico. Fisioter Calid Vida. 2011;14(1):17-9.

15. Sánchez-Ibáñez JM, Fernández ME, Moreno C, Marti D, Belón P. Ultrasound-Guided EPI ${ }^{\circledR}$ Technique and Eccentric Exercise, New Treatment for Achilles and Patellar Tendinopathy Focused on the Region-Specific of the Tendon. Orthop Muscular Syst. 2015;4(4):1-6.

16. Valera Garrido F. Short- and long-term outcomes of electrolysis percutaneous intratissue (epi) in chronic lateral elbow epicondylitis. $\mathrm{Br}$ J Sports Med. 2013;47(9):20.

17. Sánchez-Ibáñez JM, Fernández ME, Monllau JC, Alonso-Díez A, Sánchez-García, Sánchez-Sánchez JL. New treatments for degenerative tendinopathy, focused on the region-specific of the tendon. Reumatology (Sunnyvale). 2015;5(4):173. 